\title{
Twelve tips to facilitate interprofessional education and collaborative practice with students on placements in healthcare settings
}

\section{Priya Martin ${ }^{1}$ and Michael $S y^{2}$}

\begin{abstract}
It is acknowledged that the adverse effects of the COVID-19 pandemic will be long-lasting on healthcare organisations. Consequently, healthcare teams will need to work more collaboratively, supporting each other better in the post-pandemic period. Pre-registration clinical placements in healthcare settings provide an ideal opportunity to instil Interprofessional Education and Collaborative Practice (IPECP) skills, values, and competencies in students early-on. It is a common belief that teams need to have students from two or more professions to facilitate IPECP. Whilst this may be the ideal scenario, healthcare settings were struggling even prior to the pandemic to orchestrate such placement opportunities given the complicated logistics and their resource-intensive nature. This 12 tips paper provides clinical educators with practical tips to facilitate IPECP across the whole continuum from a single student on placement, to several students from two or more professions on placement at the same time. These tips, by promoting IPECP in all student placements, have the potential to re-energise IPECP in healthcare settings, thereby contributing to better outcomes for healthcare professionals, organisations, and service users.
\end{abstract}

Keywords: Interprofessional education; collaborative practice

1. Senior Research Fellow, Rural Clinical School, Faculty of Medicine, The University of Queensland, Australia

2. Associate Professor, National Teacher Training Center for the Health Professions, University of the Philippines, Manila (corresponding author)

Address for correspondence: Michael Sy mpsy@up.edu.ph

Date of first (online) publication:

69 J. of Practice Teaching \& Learning: Advance (c) wEb 


\section{Introduction}

At a time when interprofessional education and collaborative practice (IPECP) was gaining momentum internationally (Khalili et al., 2019), the COVID-19 pandemic seems to have pressured healthcare professionals to return to more siloed ways of practice due to the enormous pressures imposed on healthcare organisations (Wensing et al., 2020). Whilst an obvious impetus to re-energising IPECP is enhanced patient outcomes (Hammick et al., 2007; Reeves et al., 2008), its potential to support healthcare professionals in their roles through the pandemic and in the post-pandemic period cannot be underestimated (Khalili et al., 2021). Concerns have also been raised about the impact of the pandemic on work preparedness of graduates who will be entering the workforce over the next few years, given the disruptions to student placements that have affected authentic learning experiences (Choi et al., 2020; Syned et al., 2020; Ulenaers et al., 2021). This makes it even more vital to propagate IPECP in healthcare settings, to restore and strengthen a culture of collaborative practice, starting with students who undertake clinical placements to fulfil their course requirements.

Interprofessional education has been defined by the World Health Organisation (WHO) as an experience that occurs when students from two or more professions learn about, from, and with each other (WHO, 2010). Whereas, collaborative practice (or interprofessional collaborative practice) in healthcare occurs when multiple healthcare professionals from different disciplines provide comprehensive services by working with patients, their families, carers and communities to deliver the highest quality of care across settings (WHO, 2010). Whilst it is easier to bring students from two or more professions together in university settings to facilitate IPECP, it remains a challenge in healthcare settings, given the complex logistics of coordinating student placement schedules across disciplines and/or universities (Boet et al., 2014). This has contributed to the wide gap in the IPECP experiences of students in universities as opposed to healthcare settings (Barr et al., 2017). To bridge this gap efficiently, healthcare professionals need to capitalise on all opportunities they have with students to promote IPECP. We propose that IPECP can be facilitated across the whole continuum from a single student on placement (a realistic, more common scenario), to several students from two or more professions on placement in the same location at the same time (an idealistic, less common scenario).

70 J. of Practice Teaching \& Learning: Advance $\odot$ w $\& b$ 
The twelve tips presented in this paper are based on the available literature as well as the authors' collective experience in championing IPECP implementation, teaching and research. Whilst the first six tips target those that only have one student on placement at any given time, tips seven to ten are applicable to those facilitating IPECP for more than one student, representing two or more professions. Tips 11 and 12 are for all clinical educators (supervisors) that are interested in facilitating IPECP with students or other learners (such as interns) on placements. To alleviate any confusion in terminology, we have used the term/abbreviation IPECP consistently in this paper to incorporate both interprofessional education and interprofessional collaborative practice.

\section{The Tips}

\section{TIP 1: Embed IPECP into the placement structures and processes}

For the student to appreciate and engage actively in IPECP activities, it is important to weave IPECP competencies into the placement structures and processes. IPECP can be incorporated in the student's placement learning goals, and the competencies can be mapped on the placement assessment tool. This will enable the student to appreciate that IPECP is not a standalone 'add-on' but a well-integrated component of their placement. Frameworks such as the Canadian Interprofessional Health Collaborative (CIHC) Framework (CIHC; 2010) or the Interprofessional Education Collaborative framework (Boet et al., 2014; IPEC; 2016) are commonly used frameworks that will be suitable for this purpose. The framework chosen can also guide the development of tutorial content and case studies. Setting expectations and a plan early in the placement will better prepare the student to fully engage with IPECP activities (Sy et al., 2020). The student can be taught by other healthcare professionals in the team, carers, and even clients themselves which is likely to provide a rich learning experience that fosters IPECP in action.

71 J. of Practice Teaching \& Learning: Advance $\odot$ w Eb 


\section{TIP 2: Apply theories and frameworks to facilitate IPECP in practice}

One of the reasons for the global IPECP momentum in the past decade is the persistent co-construction of theory-informed and research-driven arguments produced by educators and researchers (Teodorczuk et al., 2016). While there is a range of theories available to inform IPECP, oftentimes these theories remain abstract concepts taught at universities and not applied in practice, contributing to the theory-practice gap. Clinical educators must digress from the idea that theory is only utilised by academics, but rather recognize that the use of theory helps in understanding and providing solutions to practical issues (Reeves \& Hean, 2013). Underpinning IPECP with sound theory does not only provide opportunities to solve modern-day issues of learning, practicing, and caring among healthcare professionals, but also offers justifications to those who are sceptical of interprofessionalism (Hean et al., 2009).

Practical ways to apply IPECP theory in placements include the use of case-based learning, simulation-based learning and the deliberate use of learning activities anchored to the International Classification of Functioning, Disability and Health (ICF). Evidence suggests that providing case studies based on real scenarios can hone students' confidence, leadership and other transferable skills that are essential for actual client care (WHO, 2010; Soubra et al., 2018). Principles of case-based learning was successfully applied in a study of 21 new graduates who participated in a six-month IPECP program at one health service in Australia, where the researchers used case-based scenarios to unpack roles of different professionals to promote IPECP (Martin et al., 2016). Involving students in case-based learning opportunities with clinical and community-focused cases (Sy, 2017) and problem-based small group discussions with tutorials can provide a safe and non-threatening space to discuss clinical cases while being aware of self and others in the team (Alinea, 2010). Boet and colleagues (2014) have outlined practical tips for clinical educators seeking to enhance IPECP through simulation-based learning. Concrete examples are available on facilitating theory-informed IPECP using the ICF framework to foster mutual understanding of health-related cases among students from different disciplines (Moran et al., 2020).

72 J. of Practice Teaching \& Learning: Advance $\odot$ w $-b$ 


\section{TIP 3: Utilise available IPECP tools}

There are several tools freely available to help the student learner and the teams hosting students to better engage with IPECP. The Centre for Interprofessional Education at the University of Toronto (2019) offers three flexible learning activities and associated resources for learners, namely: shadowing and/or interviewing team members; analysing interprofessional interactions of team members; and, collaborating with team members. These tools have been used to promote IPECP competencies for students in a recent Australian study (Martin, Hill et al., 2021; Martin, Moran et al., 2021). A student working on role clarification and interprofessional communication can undertake shadowing of a team member from another profession and use the shadowing and/or interviewing team members flexible activity tip sheet to guide them to undertake the activity proficiently. Whilst this tip sheet is said to be useful particularly to learners in early placements, they can be equally beneficial to the clinical educator as it steps them through questions that they can ask the student to facilitate reflection and debriefing following the shadowing experience. Another tool, the Points for Interprofessional Education System (PIPEs; Centre for Interprofessional Education, University of Toronto, 2019), provides a checklist, prompts, and tips to the clinical educator to make teaching content and delivery more interprofessional. The Interprofessional Collaborative Organizational Map and Preparedness Assessment (IP-COMPASS) is a tool that provides a quality improvement framework for healthcare teams and organizations to become more prepared to offer IPECP. The IP-COMPASS can enhance the understanding and awareness of the attributes of the organizational culture that can create an environment that is conducive to IPECP (Parker et al., 2012).

\section{TIP 4: Enrich IPECP at the team level}

Interprofessional teamwork, integral to IPECP 'involves different health and/or social professions who, for example, share a team identity and work closely together in an integrated and interdependent manner to solve complex care problems and deliver services' (Journal of Interprofessional Care, n.d.). Students in clinical placements can experience interprofessional teamwork when they actively participate in case conferences, ward/teaching rounds, and team meetings. For instance, in case conferences, the student

73 J. of Practice Teaching \& Learning: Advance $\odot$ wEbb 
can be given an opportunity to present a case and receive feedback from other healthcare professionals and the clinical educator. The student can be given responsibilities to assist during ward rounds as the clinical educator sees fit. Team meetings become an avenue for learning when the student can observe how practicing healthcare professionals take turns in reporting observations to the team, foster two-way communication (including agreement and disagreement), make team-based decisions, and act as a team (Sy, 2017). Kassutto and colleagues (2020) have provided several practical tips for healthcare teams to make teaching rounds on the ward more interprofessional.

\section{TIP 5: Expand IPECP beyond the team level}

Although interprofessional teamwork is mostly situated in smaller and closely-knit teams characterised by a shared team identity, interprofessional collaboration provides an opportunity to work together across teams in a less integrated and interdependent manner (Journal of Interprofessional Care, n. d.). In clinical placements, IPECP occurs when teams from the same department or unit work together towards providing quality care to clients. This can also be extended when two or three teams from different units gather to develop an integrated plan for a complex case in the ward or a community-based health service facility. Notably, IPECP is distinct from interprofessional teamwork because a cohort of healthcare and social welfare workers are working together to plan, manage, and deliver care services. For instance, during discharge planning, a medical student and a nursing student on an acute ward may need to liaise with the community pharmacist and social worker regarding prescription medicines to be taken home and social welfare benefits that can be claimed by the patient upon discharge, respectively. Such collaboration across teams, can also bridge the hospital-primary care divide, to provide holistic care that is more meaningful, relevant, and contextual to clients and communities (Ensing et al., 2015).

\section{TIP 6: Role model collaborative and client-centred practice}

In the absence of other student learners, IPECP for the solo student on placement, relies heavily on healthcare professionals within the team.

74 J. of Practice Teaching \& Learning: Advance (C) wEb 
Workplace modelling is a powerful tool to promote IPECP (Page et al., 2009). In clinical pharmacy education, role modelling is perceived as an opportunity to employ vicarious learning and to facilitate mentorship between learners and educators (Page et al., 2009). In other studies, role modelling was seen as a way to teach affective competencies such as valuing and respecting others' professions (Croker et al., 2016), effective working within a healthcare team (Walker et al., 2019), and leadership (Hu \& Broome, 2020). Such competencies can be concretely translated by allowing students to learn from professionals from varied backgrounds. For instance, medical students involved in stroke rehabilitation may be supervised by or receive feedback from an occupational therapy educator to activate patient advocacy roles (O'Leary \& Cantillon, 2021). Alternatively, in a case discussion involving a nutrition and dietetic student, a speech pathology educator may provide a short lecture about dysphagia management. Work shadowing between different professions (e.g., medical students observing a nurse practitioner during cannulation) and between professionals and clients (e.g., medical students observing how an occupational therapist provides feedback to parents of a child with additional needs) are some other ways of role modelling IPECP. Aside from modelling best practice in client- and family-centred care, these strategies can also offer a different lens of understanding a case outside their uni-professional reasoning, which better prepares students and interns for practice in the real world (O'Leary \& Cantillon, 2021) and fosters development of their interprofessional identity (Tong et al., 2021).

\section{TIP 7: Consider logistics}

It is well-acknowledged that interprofessional student placements are hard to achieve due to the complex logistical considerations involved in coordinating placement schedules across professions (Boet et al., 2014). Liaising with those that hold responsibilities in coordinating student placements from different professions or identified IPECP champions, may provide an indication of the best periods of overlap between learners from different professions. An Australian study reports an interprofessional model of student placement, the Rural Interprofessional Education and Supervision (RIPES) model, where students from two or more professions undertake their placements in a given healthcare setting concurrently, with an overlapping period of five weeks. This period is used to promote IPECP

75 J. of Practice Teaching \& Learning: Advance $\odot$ wEb 
in students through tailored activities such as weekly interprofessional skills sessions, structured work shadowing, peer learning, and joint client sessions (Martin, Hill et al., 2021; Martin, Moran et al., 2021). Although such a level of coordination is resource-intensive and hard to achieve, evaluation results of the study have revealed several benefits of the RIPES model to students, clinical educators, and workplaces. The model also produced ripple effects in participating teams by further embedding IPECP in service delivery post-placement, making the resource-investment worthwhile (Martin, Hill et al., 2021).

Co-location of the student workspaces, when there are two or more students, is recommended practice as co-location can enhance collaborative practice and patient outcomes (Bonciani et al., 2018; Carney et al., 2019). It can also be useful to plan and book dedicated spaces in advanced where IPECP tutorials, teaching or case discussions can be held with students physically or virtually so that IPECP is well-planned and executed throughout the placement as discussed in Tip 1.

\section{Tip 8: Promote joint client sessions}

Healthcare professionals undertaking joint client sessions can better streamline client care. A randomised controlled trial of joint consultation sessions between general practitioners and specialists found that this approach resulted in more efficient care as well as better targeted examination, treatment, and referrals (Vierhout et al., 1995). When a healthcare team uses an IPECP model of care, it can be easier to identify opportunities where the students can observe and participate in joint client sessions. This may take the form of healthcare professionals from two or more professions undertaking a client assessment, therapy, or intervention at the same time in a joint manner. For instance, a physician and a nurse on an acute in-patient ward may undertake assessment of a client's pressure sore together to make a joint plan for intervention. A physiotherapist and an occupational therapist may jointly ambulate a client around the hospital ward to determine their capacity and readiness to participate in a predischarge home visit. When the clinical educator takes part in these joint client sessions, they are role modelling interprofessional communication, collaborative practice, and client-centred care to the student, as outlined in Tip 6. Such experiences enable the student to connect all the dots and understand the patient's journey in relation to different professionals that are

76 J. of Practice Teaching \& Learning: Advance (C) wEb 
involved in the care delivery. Students can also be encouraged to organise and participate in joint client sessions as applicable and appropriate with the required amount of supervision provided by the clinical educator. Making time to debrief the students after such joint client sessions can reinforce students' reflective learning and critical thinking. For teams that do not currently use an IPECP model of care, advanced planning of such sessions or utilising newer services/clinics to build in joint client sessions, can help overcome the scheduling hurdles.

\section{TIP 9: Orchestrate peer-assisted learning amongst students}

Peer-assisted learning in clinical settings occurs between students on placement directly (e.g., tutorials) and indirectly (e.g., peer observation and feedback on the ward) and can be more useful if there is an educator present to moderate and guide the learning (Tai et al., 2017). Clinical educators can create opportunities for students to undertake learning together and help to moderate discussions about quality performance (Tai et al., 2017). For instance, students can be provided with protected time to engage in peer-assisted learning where a more senior student can assist a more junior learner. Another illustration can be a student who has undertaken shadowing using the University of Toronto Flexible Learning Activity Tip Sheet (see Tip 3) may first reflect on the experience with a peer prior to debriefing with the clinical educator. This will not only promote learning with peers but can improve the confidence of students when debriefing with the clinical educator and subsequently lighten the clinical educator's workload. Co-location of student workspaces (see Tip 8) can facilitate indirect/informal peer-assisted learning amongst students.

\section{TIP 10: Encourage student-led project and presentation}

Students presenting their project or a case study to the host team can foster several IPECP competencies including interprofessional communication, teamwork, and collaborative leadership. In the RIPES model of student placement (Martin, Hill et al., 2021, see Tip 7), students collaborated to complete a small-scale project during the five weeks of placement. This IPECP-focused project was chosen by the students at the start of the placement in conjunction with the clinical educator. This provided

77 J. of Practice Teaching \& Learning: Advance $\odot$ w Eb 
students an opportunity to assess the IPECP structures and processes at the placement site, identify strengths and areas for improvement, and develop resources or a process to fill an existing gap. In the RIPES study, students were given an option to work on a case study in lieu of the project, which was presented at the conclusion of the placement.

\section{TIP 11: Undertake targeted professional development to acquire skills, knowledge, and confidence in IPECP and facilitation of IPECP}

Continuing professional development helps healthcare professionals acquire the knowledge, skills, confidence, and attitudes necessary to create and sustain a culture of collaboration and ultimately improves quality care and patient safety (Lown et al., 2011). Activities towards professional development can include continuing education (e.g., completing formal courses and degree programs, certifications, and credentialing), attending and/or presenting in conferences, participating and/or facilitating workshops, volunteering in committees, research, job performance enhancement and undertaking independent projects. Facilitating IPECP in learners starts with the educator first upskilling in this area (Sy et al., 2021; Lie et al., 2016), which helps with facilitating an evidence-informed and intentional IPECP placement. Clinical educators can give the student role assignments in the unit, offer to join a committee for a short-term task, and, when able, allow them to lead a team (thereby, fostering collaborative/ distributed leadership). Evaluating IPECP competencies along with uniprofessional competencies will provide students with valuable feedback on their clinical skills, as well as transferable skills that are anchored on interprofessional competencies (Buring et al., 2009).

\section{TIP 12: Evaluate IPECP initiatives in placements}

In line with best practice principles, it is recommended that clinical educators and teams evaluate IPECP opportunities and initiatives involving students and other learners (Boet et al.,2014). This can be done informally during supervision sessions, with a view of using the feedback gathered to improvise future IPECP initiatives. Alternatively,

78 J. of Practice Teaching \& Learning: Advance (C) wEb 
clinical educators interested in formally researching IPECP initiatives can be guided by the practical tips outlined in recent publications (Khalili et al., 2019; O'Carroll et al., 2020). It is recommended that IPECP research teams consist of at least one researcher from the university, co-educator(s), and service users if deemed fitting (O'Carroll et al., 2020). For quantitative methods, commonly reported survey tools found on the National Center for Interprofessional Practice and Education website can be used (URL: https://nexusipe.org/advancing/assessment-evaluationstart). For qualitative methods, interviews and focus groups can be used (El-Awaisi et al., 2018; Ding et al., 2020). Using a combination of methods (i.e., quantitative and qualitative), is recommended to ensure that the IPECP opportunities for students are robust and fit-for-purpose in promoting the necessary skills, knowledge and confidence in student learners, by answering the what, how, why and so what questions. The evaluation or research data can then be used for requests linked to return on investment or grant funding (Boet et al., 2014).

\section{Conclusion}

Whilst student placement models involving students from two or more professions are ideally placed to facilitate IPECP, many healthcare settings are unable to navigate the scheduling hurdles and resource investments to create such placements opportunities. Given the additional pressures and resource constraints induced on healthcare organisations by the COVID-19 pandemic, it is imperative that clinical educators and healthcare teams are prepared to facilitate IPECP even when there is a single student on placement. We have developed these tips for clinical educators and teams to use across a continuum of student placement models, from singular students to a group of students from two or more professions. Doing so will generate further interest in facilitating IPECP in all student placement scenarios, which will be crucial in re-energising IPECP models of care and collaborative working in healthcare organisations in the post-pandemic period.

79 J. of Practice Teaching \& Learning: Advance $\odot$ w Eb 


\section{References}

Alinea, M. C. D. (2010) Strategies in teaching small groups. in E. A. Sana (Ed.) Teaching and learning in the health sciences. Quezon City: University of the Philippines Press. (pp.107-128)

Barr, H., Ford, J., Gray, R., Helme, M., Hutchings, M., Low, H., Machin, A. and Reeves, S. (2017) Interprofessional education guidelines 2017. Centre for the Advancement of Interprofessional Education (CAIPE). [Accessed 12 August 2021 at https://www.caipe.org/resources/publications/caipe-publications/ caipe-2017-interprofessional-education-guidelines-barr-h-ford-j-gray-r-helmem-hutchings-m-low-h-machin-reeves-s]

Boet, S., Bould, M. D., Layat Burn, C., and Reeves, S. (2014) Twelve tips for a successful interprofessional team-based high-fidelity simulation education session. Medical teacher, 36, 10, 853-857 [Accessed 12 August 2021 at https:// doi.org/10.3109/0142159X.2014.923558]

Bonciani, M., Schäfer, W., Barsanti, S., Heinemann, S., and Groenewegen, P. P. (2018) The benefits of co-location in primary care practices: the perspectives of general practitioners and patients in 34 countries. BMC health services research, 18, 1, 132 [Accessed 12 August 2021 at https://doi.org/10.1186/ s12913-018-2913-4]

Buring, S. M., Bhushan, A., Broeseker, A., Conway, S., Duncan-Hewitt, W., Hansen, L., and Westberg, S. (2009) Interprofessional education: definitions, student competencies, and guidelines for implementation. American journal of pharmaceutical education, 73, 4, 59 [Accessed 12 August 2021 at https://doi. org/10.5688/aj730459]

Carney, P. A., Thayer, E. K., Palmer, R., Galper, A. B., Zierler, B., and Eiff, M. P. (2019) The benefits of interprofessional learning and teamwork in primary care ambulatory training settings. Journal of interprofessional education $\&$ practice, 15, 119-26. [Accessed 12 August 2021 at https://doi.org/10.1016/j. xjep.2019.03.011]

Centre for Interprofessional Education, University of Toronto. (2019). General curriculum: Materials/resources. [Accessed 12 August 2021 https://ipe.utoronto. $\mathrm{ca} /$ curriculum/facilitators/tools-resources]

Choi, B., Jegatheeswaran, L., Minocha, A., Alhilani, M., Nakhoul, M. and Mutengesa, E. (2020) The impact of the COVID-19 pandemic on final year medical students in the United Kingdom: a national survey. BMC Medical education, 20, 206. [Accessed 12 August 2021 https://doi.org/10.1186/s12909020-02117-1]

Canadian Interprofessional Health Collaborative. (2010) A national interprofessional

80 J. of Practice Teaching E Learning: Advance (C) wEb 
Twelve tips for interprofessional education and collaboration in healthcare placements

competency framework. [Accessed 12 August 2021 http://www. cihc.ca/ resources/publications]

Croker, A., Smith, T., Fisher, K., and Littlejohns, S. (2016) Educators' Interprofessional Collaborative Relationships: Helping Pharmacy Students Learn to Work with Other Professions. Pharmacy (Basel, Switzerland), 4, 2, 17 [Accessed 12 August 2021 https://doi.org/10.3390/pharmacy4020017]

El-Awaisi, A., El Hajj, M. S., Joseph, S., and Diack, L. (2018) Perspectives of practising pharmacists towards interprofessional education and collaborative practice in Qatar. International journal of clinical pharmacy, 40, 5, 1388-1401 [Accessed 12 August 2021 https://doi.org/10.1007/s11096-018-0686-9]

Ensing, H. T., Koster, E. S., Stuijt, C. C., van Dooren, A. A., and Bouvy, M. L. (2015) Bridging the gap between hospital and primary care: the pharmacist home visit. International journal of clinical pharmacy, 37, 3, 430-434 [Accessed 12 August 2021 https://doi.org/10.1007/s11096-015-0093-4]

Hammick, M., Freeth, D., Koppel, I., Reeves, S., and Barr, H. (2007) A best evidence systematic review of interprofessional education: BEME Guide no. 9. Medical teacher, 29, 8, 735-751 [Accessed 12 August 2021 https://doi. org/10.1080/01421590701682576]

Hean, S., Craddock, D. and O'Halloran, C. (2009) Learning theories and interprofessional education: A user's guide. Learning in health and social care, 8 , 4, 250-262 [Accessed 12 August 2021 doi:10.1111/j.1473-6861.2009.00227.x]

$\mathrm{Hu}, \mathrm{Y}$. and Broome, M. (2020) Leadership characteristics for interprofessional collaboration in China. Journal of professional nursing: official journal of the American Association of Colleges of Nursing, 36, 5, 356-363 [Accessed 12 August 2021 https://doi.org/10.1016/j.profnurs.2020.02.008]

Interprofessional Education Collaborative. (2016) Core competencies for interprofessional collaborative practice: 2016 update. Washington, DC: Interprofessional Education Collaborative.

Kassutto, S., Seam, N., Carlos, W. G., Kelm, D., Kaul, V., Stewart, N. H., and Hinkle, L. (2020) Twelve tips for conducting successful interprofessional teaching rounds. Medical teacher, 42, 1, 24-29 [Accessed 12 August 2021 https://doi.or g/10.1080/0142159X.2018.1545086]

Khalili, H., Lising, D., Gilbert, J. Thistlethwaite, J., Pfeifle, A., Maxwell, B., Başer Kolcu, I., Langlois, S., Najjar, G., MacMillan, Al-Hamdan, Z., K., Schneider, C., Kolcu, G., El-Awaisi, A., Ward, H., and Rodrigues, F. J. (2021) Building Resilience in Health Care in the time of COVID-19 through Collaboration - A Call to Action. InterprofessionalResearch.Global [Accessed 12 August 2021 https:// interprofessional.global/the-interprofessionalresearch-global-the-globalnetwork-for-interprofessional-education-and-collaborative-practice-research-

81 J. of Practice Teaching \& Learning: Advance $\odot$ w Eb 
has-published-building-resilience-in-health-care-in-the-time-of-covid-19/] Khalili, H., Thistlethwaite, J., El-Awaisi, A., Pfeifle., A., Gilbert, J., Macmillan, K., et al. (2019) Guidance on global interprofessional education and collaborative practice research: discussion paper. Interprofessional Research Global [Accessed 12 August 2021 https://doi.org/10.21256/zhaw-19793]

Lie, D. A., Forest, C. P., Kysh, L., and Sinclair, L. (2016) Interprofessional education and practice guide No. 5: Interprofessional teaching for prequalification students in clinical settings. Journal of interprofessional care, 30, 3, 324-330 [Accessed 12 August 2021 https://doi.org/10.3109/13561820.2016.1141752]

Lown, B. A., Kryworuchko, J., Bieber, C., Lillie, D. M., Kelly, C., Berger, B., and Loh, A. (2011) Continuing professional development for interprofessional teams supporting patients in healthcare decision making. Journal of interprofessional care, 25, 6, 401-408 [Accessed 12 August 2021 https://doi.org/10.3109/1356 1820.2011.583563]

Martin, P., Moran, M., Graham, N., and Hill, A. (2021) The Integral Role of Organisational Governance in Promoting Interprofessional Education in Rural Settings. International Journal of Environmental Research and Public Health, 18, 6, 3041. [Accessed 12 August 2021 https://doi.org/10.3390/ijerph18063041]

Martin, P., Hill, A., Graham, N., Argus, G., and Ford M. (2021) Sustaining interprofessional initiatives in the current landscape: A short report on interprofessional education in rural health services in Queensland. Australian Journal of Rural Health, 29, 2, 245-247 [Accessed 12 August 2021https://doi. org/10.1111/ajr.12714]

Martin, P., Newby, M., Moran, M., Browne, M., and Kumar, S. (2016) Interprofessional Scenario-Based Learning for New Graduates in a Regional Setting: A Pilot Study. The internet journal of allied health sciences $\&$ practice, 14, 3. [Accessed 12 August 2021http://nsuworks.nova.edu/ijahsp/vol14/ iss3/6/?utm_source=nsuworks.nova.edu\%2Fijahsp\%2Fvoll4\%2Fiss3\%2F6\& utm_medium=PDF\&utm_campaign=PDFCoverPages]

National Center for Interprofessional Practice and Education. (2021) Assessment \& evaluation. [Accessed 12 August 2021 https://nexusipe.org/advancing/ assessment-evaluation-start]

O'Carroll, V., Owens, M., Sy, M., El-Awaisi, A., Xyrichis, A., Leigh, J., Nagraj, S., Huber, M., Hutchings, M., and McFadyen, A. (2020) Top tips for interprofessional education and collaborative practice research: a guide for students and early career researchers. Journal of interprofessional care, 35, 3, 328-333 [Accessed 12 August 2021 https://doi.org/10.1080/13561820.2020.1777092]

O'Leary, N. and Cantillon, P. (2021) Longitudinal integrated clerkships are ok, but do they prepare students for reality?. Medical education, 55, 5, 544-546

82 J. of Practice Teaching $\&$ Learning: Advance $\odot$ w $\mathbb{E} b$ 
Twelve tips for interprofessional education and collaboration in healthcare placements

[Accessed 12 August 2021 https://doi.org/10.1111/medu.14454]

Page, R., Hume, A., Trujillo, J., Leader, W., Vardeny, O., Neuhauser, M., Dang, D., Nesbit, S., and Cohen, L.J. (2009) Interprofessional Education: Principles and Application A Framework for Clinical Pharmacy. Pharmacotherapy: The Journal of Human Pharmacology and Drug Therapy, 29, 7, 879-879 [Accessed 12 August 2021 https://doi.org/10.1592/phco.29.7.879]

Parker, K., Jacobson, A., McGuire, M., Zorzi, R., and Oandasan, I. (2012) How to build high-quality interprofessional collaboration and education in your hospital: the IP-COMPASS tool. Quality management in health care, 21, 3, 160-168 [Accessed 12 August 2021 https://doi.org/10.1097/QMH.0b013e31825e87a2]

Reeves, S. and Hean, S. (2013). Why we need theory to help us better understand the nature of interprofessional education, practice and care. Journal of interprofessional care, 27, 1, 1-3 [Accessed 12 August 2021 https://doi.org/10.3 109/13561820.2013.751293]

Reeves, S., Zwarenstein, M., Goldman, J., Barr, H., Freeth, D., Hammick, M., and Koppel, I. (2008) Interprofessional education: effects on professional practice and health care outcomes. The Cochrane database of systematic reviews, 1, CD002213 [Accessed 12 August 2021 https://doi.org/10.1002/14651858. CD002213.pub2]

Soubra, L., Badr, S. B. Y., Zahran, E. M., and Aboul-Seoud, M. (2018) Effect of Interprofessional Education on Role Clarification and Patient Care Planning by Health Professions Students. Health professions education, 4, 4, 317-328 [Accessed 12 August 2021 https://doi.org/10.1016/j.hpe.2017.12.005]

Sy, M. P. (2017) Filipino therapists' experiences and attitudes of interprofessional education and collaboration: A cross-sectional survey. Journal of interprofessional care, 31, 6, 761-770 [Accessed 12 August 2021 https://doi.org/10.1080/13561 820.2017.1359509]

Sy, M., Pineda, R. C., Sumulong, R. A., and Navarro, A. (2020) Establishing a Pilot Interprofessional Education (IPE) Program in a Higher Education Institution (HEI) in the Philippines. Journal of health and caring sciences, 2, 2, 180-191 [Accessed 12 August 2021 https://doi.org/10.37719/jhcs.2020.v2i2.rna004]

Tai, J. H., Canny, B. J., Haines, T. P., and Molloy, E. K. (2017). Identifying Opportunities for Peer Learning: An Observational Study of Medical Students on Clinical Placements. Teaching and learning in medicine, 29, 1, 13-24 [Accessed 12 August 2021 https://doi.org/10.1080/10401334.2016.1165101]

Teodorczuk, A., Khoo, T. K., Morrissey, S., and Rogers, G. (2016) Developing interprofessional education: putting theory into practice. The clinical teacher, 13, 1, 7-12 [Accessed 12 August 2021 https://doi.org/10.1111/tct.12508]

Tong, R., Brewer M., Flavell, H. and Roberts, L. D. (2021). Facilitating

83 J. of Practice Teaching \& Learning: Advance $\odot$ w\&b 
interprofessional identity development in healthcare students through dedicated interprofessional placements. Journal of interprofessional care [Accessed 12 August 2021 https://doi.org/10.1080/13561820.2021.1883564]

Ulenaers, D., Grosemans, J., Schrooten, W., and Bergs, J. (2021) Clinical placement experience of nursing students during the COVID-19 pandemic: A cross-sectional study. Nurse education today, 99, 104746 [Accessed 12 August 2021https://doi.org/10.1016/j.nedt.2021.104746]

Vierhout, W. P., Knottnerus, J. A., van OOij, A., Crebolder, H. F., Pop, P., Wesselingh-Megens, A. M., and Beusmans, G. H. (1995) Effectiveness of joint consultation sessions of general practitioners and orthopaedic surgeons for locomotor-system disorders. Lancet (London, England), 346, 8981, 990-994. [Accessed 12 August 2021 https://doi.org/10.1016/s0140-6736(95)91686-5]

Walker, L. E., Cross, M., and Barnett, T. (2019) Students' experiences and perceptions of interprofessional education during rural placement: A mixed methods study. Nurse education today, 75, 28-34. [Accessed 12 August 2021 https://doi.org/10.1016/j.nedt.2018.12.012]

Wensing, M., Sales, A., Armstrong, R., and Wilson, P. (2020) Implementation science in times of Covid-19. Implementation science, 15, 1, 42. [Accessed 12 August 2021 https://doi.org/10.1186/s13012-020-01006-x]

World Health Organisation. (2010) Framework for action on interprofessional education and collaborative practice. Geneva: World Health Organisation [Accessed 12 August 2021 https://www.who.int/publications/i/item/ framework-for-action-on-interprofessional-education-collaborative-practice]

84 J. of Practice Teaching \& Learning: Advance (C) wEb 